\title{
Mehrskalenberechnungen nichtlinear elastischer inhomogener Körper
}

\author{
Johann Bitzenbauer ; Karl Schweizerhof
}

\begin{abstract}
Mehrgittermethoden als besonders schnelle und effiziente Lösungsverfahren für elliptische partielle Differentialgleichungen basieren auf der Diskretisierung des Problems mittels unterschiedlich grober bzw. feiner Netze. Ihre Effizienz leidet jedoch sehr, sobald eine sehr grobe - aber immer noch problemadäquate - Diskretisierung aufgrund vorhandener komplexer Ränder oder materieller Inhomogenitäten nicht mehr möglich ist. Andererseits führt aber jede klassische FE-Analyse, die komplizierte geometrische Details befriedigend auföst, zu so vielen Unbekannten, dass nicht multilevelbasierte Gleichungslöser von vorneherein ausscheiden. In [1] werden deshalb Finite-Elemente-Räume entwickelt, deren minimale Dimension nicht mehr mit der Komplexität der Geometrie gekoppelt ist. Diese sogenannte zusammengesetzte Finiten-ElementeDiskretisierung (CFE) wird über eine Gitterhierarchie definiert. In [2] wird das Konzept auf materiell inhomogene Probleme erweitert. Im Vergleich zu rein algebraisch erzeugten niederdimensionalen Problemen werden bei diesem Vorgehen zusätzliche Geometrieinformationen genutzt. Für einfache eindimensionale Problemklassen besteht kein prinzipieller Unterschied zwischen CFE- und algebraisch motivierten Methoden; dies ändert sich jedoch bei den hier betrachteten zwei- bzw. dreidimensionalen Problemen der nichtlinearen Elastizitätstheorie. Die Konstruktion der Interpolationsoperatoren erfordert zunächst eine hierarchische Schachtelung der Gitter. Ist diese nicht vorhanden, z.b. weil eine Diskretisierung bereits vorgegeben ist, können allerdings auch nachträglich unter Verwendung einer Quadtree/Octree-Datenstruktur [3] passende Hilfsgitter konstruiert werden [4]. Ein solcher Algorithmus entspricht dann sozusagen einem hybrid geometrisch-algebraischen Mehrgitterverfahren. Bestehende Algorithmen, die für mittels Dreieckselementen diskretisierte Gleichungen vom Poisson-Typ entwickelt wurden, werden im Beitrag auf zwei- und dreidimensionale nichtlinear elastische inhomogene Körper erweitert. Deren Triangulierungen können nun sowohl aus Drei- und Viereckselementen bzw. Tetraedern und Hexaedern bestehen. Konvergenzstudien für uniform und adaptiv verfeinerte Gitter zeigen an ausgewählten Beispielen für inhomogene elastische Körper mit großen Deformationen die Robustheit und Leistungsfähigkeit der Methode.
\end{abstract}

[1] Sauter, S.A. [1997]: Vergröberung von Finite-Elemente-Räumen. Habilitation, Universität Kiel.

[2] Warnke, R. [2003]: Schnelle Löser für elliptische Randwertprobleme mit springenden Koeffizienten. Dissertation, Universität Zürich.

[3] Samet, H. [1990]: The Design and Analysis of Spatial Data Structures. Addison-Wesley.

[4] Feuchter, D.; Heppner, I.; Sauter, S.A.; Wittum, G. [2003]: Bridging the 
gap between geometric and algebraic multi-grid methods. Computation and Visualization in Science 6, pp. 1-13. 